[Article]

\title{
异辛烷/正庚烷/乙醇三组分燃料着火的化学动力学模型
}

\author{
郑东钟北京* \\ (清华大学航天航空学院, 北京 100084)
}

\begin{abstract}
摘要: 提出一个包括异辛烷、正庚烷和乙醇的三组分燃料的着火动力学模型, 该机理包括 50 个组分和 193 个 反应. 通过路径分析和灵敏度分析, 给出了基础燃料在高低温条件下的不同反应路径和影响氧化过程的重要基 元反应. 该机理预测的单组分 (异辛烷、正庚烷、乙醇)燃料、双组分基础燃料和三组分燃料的点火延迟时间与实 验值有很高一致性. 本文机理包含较少的组分数与反应数, 因而可适用汽油掺烧乙醇的多维计算流体动力学 (CFD)数值模拟.
\end{abstract}

关键词：替代燃料；基础燃料；着火延迟时间；机理； 乙醇

中图分类号: 0643

\section{Chemical Kinetic Model for Ignition of Three-Component Fuel Comprising iso-Octane/n-Heptane/Ethanol}

\author{
ZHENG Dong ZHONG Bei-Jing* \\ (School of Aerospace, Tsinghua University, Beijing 100084, P. R. China)
}

\begin{abstract}
A reduced chemical kinetic mechanism for the oxidation of three-component fuel comprising iso-octane/n-heptane/ethanol has been developed. The mechanism consists of 50 species and 193 elementary reactions and emphasizes the ignition process. Using path and sensitivity analyses, the path of primary reference fuel (PRF) oxidation and major elementary reactions at high and low temperatures are given. The validated results show that the present mechanism gives good agreement with experimental data for ignition delay time predictions. Because of the few species and reactions presenting in the chemical kinetic model, the mechanism is applicable to multidimensional computational fluid dynamic (CFD) simulation of the co-combustion of gasoline with ethanol.
\end{abstract}

Key Words: Surrogate fuel; Primary reference fuel; Ignition delay time; Mechanism; Ethanol

\section{1 引言}

随着化石燃料的日渐贵乏和环境问题的日益 尖锐, 替代燃料和新型的燃烧方式成为能源动力领 域研究的主流. 乙醇作为一种可再生的含氧生物替 代燃料, 以其良好的燃烧和排放性能备受青睐. 其 中汽油掺烧乙醇广受关注, 表 1 为乙醇和汽油特性 的对比. '可以看出乙醇含氧量较高, 有利于降低 $\mathrm{CO}$ 、未燃烧碳氢化物和碳烟等污染物的排放. 许多
研究表明, ${ }^{2-6}$ 汽油添加乙醇燃烧能够改善其污染物 排放特性. Lemaire 等 ${ }^{4}$ 的实验表明, 汽油添加 $10 \%-30 \%$ 的乙醇可以使碳烟前驱物和碳烟总量减 少 $25 \%-81 \%$. 宋崇林等 ${ }^{5}$ 的研究显示, 汽油掺烧乙醇 后苯排放降低, 并且随着添加比例的增大, 苯排放 改善更明显, 掺烧体积分数为 $9.8 \%$ 的乙醇对苯排放 的改善程度达 $50 \%$. 乙醇的辛烷值较高, 汽油内燃 机添加乙醇后可以进一步提高压缩比, 使得热效率

Received: May 2, 2012; Revised: July 4, 2012; Published on Web: July 4, 2012.

"Corresponding author. Email: zhongbj@tsinghua.edu.cn; Tel: +86-10-62772928.

The project was supported by the National Natural Science Foundation of China (51036004).

国家自然科学基金(51036004)资助项目

(C) Editorial office of Acta Physico-Chimica Sinica 
表 1 乙醇和汽油特性的对比

Table 1 Comparison of gasoline and ethanol properties

\begin{tabular}{lccccccc}
\hline & Chemical formula & Molecular weight & $w_{\text {oxygen }} / \%$ & Net lower heating value $/\left(\mathrm{MJ} \cdot \mathrm{kg}^{-1}\right)$ & Latent heat $/\left(\mathrm{kJ} \cdot \mathrm{L}^{-1}\right)$ & $\mathrm{MON}$ & $\mathrm{RON}$ \\
\hline gasoline & $\mathrm{C}_{4}-\mathrm{C}_{12}$ & $100-105$ & $0-4$ & 43.5 & 223.2 & $82-92$ & $91-100$ \\
ethanol & $\mathrm{C}_{2} \mathrm{H}_{5} \mathrm{OH}$ & 46 & 34.7 & 27 & 725.4 & 92 & 111 \\
\hline
\end{tabular}

MON: motor octane number; RON: research octane number

提高. 要实现汽油掺烧乙醇的数值模拟, 就需要提 出包含汽油和乙醇的多组分燃料机理. 由于汽油是 包含上千种燃料的混合物, 且其成分也因产地不同 而有所差异, 因此需要找到一种或者几种纯组分燃 料替代汽油, 并且能够很好地再现汽油的燃烧特 性. 基础燃料(PRF), 即异辛烷和正庚烷两组分的混 合燃料, 普遍用来代替不同辛烷值的汽油, 在内燃 机数值模拟中得到广泛的应用. ${ }^{7}$ 目前常用的基础燃 料的反应机理概况如表 2 所示..$^{8-13}$

$\mathrm{Sh} \mathrm{ll}^{8}$ 和 $\mathrm{Cox}^{9}$ 等经验模型只能描述着火前阶段 的情况, 无法反映中间产物变化和整个燃烧过程. Tanaka 简化模型 ${ }^{10}$ 虽然基本上可以描述整个燃烧过 程, 但是高温反应用 4 个总包反应直接生成 $\mathrm{CO}$ 、 $\mathrm{H}_{2} \mathrm{O} 、 \mathrm{H}_{2} \mathrm{O}_{2}$ 和 $\mathrm{HO}_{2}$, 忽略了大分子的高温裂解过程, 难以预测燃烧过程的排放特性. Tsurushima ${ }^{11}$ 给出了 一个骨架机理, 其低温反应来自于 Tanaka 简化模 型, 并添加和修正了部分反应, 这样在一定程度上 考虑了大分子高温裂解. Ra 简化模型 ${ }^{12}$ 是在 Curran 详细机理 ${ }^{13}$ 基础上分析简化得到的, 此模型能够较 为准确地计算着火延迟时间、缸压、释热率. Curran 等 ${ }^{13}$ 给出了一个非常详细的机理, 能够很好地描述 着火燃烧过程.

描述汽油燃烧的多组分反应机理也有少量研 究, 如 Andra $\mathrm{e}^{14}$ 提出的包括异辛烷、正庚烷、乙醇、甲 苯、二异丁烯五组分混合燃料的详细机理(1121 组 分、4961 反应)和简化机理 ${ }^{15}$ (142 组分、672 反应). 包 括异辛烷、正庚烷、甲苯的三组分燃料(TRF)也常作 为汽油替代燃料. 目前常用的 TRF 机理有 Andrae 等 提出的详细机理 ${ }^{16}$ 和简化机理 ${ }^{17}$, Anderlohr 等发展
的模型, ${ }^{18}$ Sakai 等提出的模型 ${ }^{19}$ 和张等人提出的模 型. ${ }^{20}$ 其中张等人提出的 TRF 模型 ${ }^{20}$ 较小, 包括 70 组 分、196反应. 由于五组分机理组分数和反应数都比 较大, 不太适用于计算流体动力学(CFD)的耦合计 算, 而 TRF 机理不包含乙醇. 因此, 本文在考虑汽油 掺烧乙醇时, 在上述基础燃料的基础上通过增加乙 醇的反应, 首先提出了包含较少组分和反应的三组 分反应动力学机理, 然后通过与实验结果的比较对 该机理进行了验证.

\section{2 三组分燃料机理的构建}

\section{1 三组分燃料机理的初步构建}

在构建异辛烷/正庚烷/乙醇三组分燃料机理 时, 首先确定基础燃料的反应机理. 在基础燃料选 择时, 不仅要考虑机理的准确性, 还要考虑机理规 模的大小. 虽然 Curran 详细机理 ${ }^{13}$ 能够较为准确地 预测燃烧特性和中间产物, 但是由于机理过于庞 大, 难以用于 CFD 耦合计算. Ra机理 ${ }^{12}$ 虽然规模较 小, 仅包含 41 组分和 130 反应, 但该机理能较为准 确地计算着火延迟时间、缸压、释热率, 而且包含较 多的 $\mathrm{C}_{2}-\mathrm{C}_{4}$ 反应, 能够反映燃烧过程中小碳分子的 变化. 因此, 本文初步选择 $\mathrm{Ra}$ 的反应机理作为基础 燃料的机理. 本文的基础燃料命名按照常用 PRF 加 辛烷值的方式, 例如 PRF60, 指基础燃料中异辛烷和 正庚烷体积分数分别是 $60 \%$ 和 $40 \%$.

其次, 我们通过对 Marinov 详细乙醇机理 ${ }^{21}$ 进行 反应路径分析, 如图 1 所示, 取节点上组分的相关反 应, 构成了乙醇部分反应机理. 由于在 $\mathrm{Ra}$ 机理中包 含了 $\mathrm{C}_{2} \mathrm{H}_{4} 、 \mathrm{CH}_{3} 、 \mathrm{CH}_{2} \mathrm{CO}$ 等小分子反应, 所以在乙醇

表 2 基础燃料(PRF)机理

Table 2 Primary reference fuel (PRF) mechanisms

\begin{tabular}{cccccccc}
\hline Number & Name & Species & Reaction & $T / \mathrm{K}$ & $p / \mathrm{MPa}$ & Equivalence ratio & Reference \\
\hline 1 & Shell & 5 & 8 & $650-850$ & $1.0-3.0$ & $0.5-1.4$ & 8 \\
2 & Cox & 10 & 15 & $650-900$ & $1.0-3.0$ & $0.5-1.4$ & 9 \\
3 & Tanaka & 32 & 55 & $750-900$ & $3.0-5.0$ & $0.2-0.6$ & 10 \\
4 & Tsurushima & 33 & 38 & $800-1500$ & $0.65-4.0$ & $0.5-2.0$ & 11 \\
5 & Ra & 41 & 130 & $700-1200$ & 4.0 & $0.5-2.0$ & 12 \\
6 & Curran & 990 & 4060 & $550-1700$ & $0.1-4.2$ & $0.3-1.5$ & 13 \\
\hline
\end{tabular}




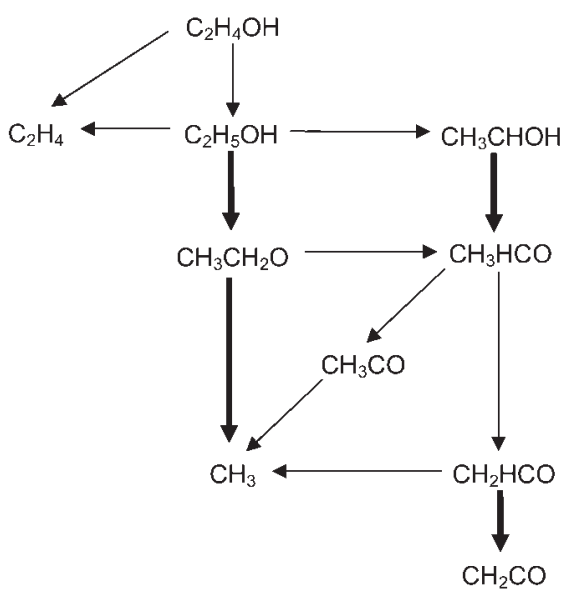

图 1 乙醇氧化部分反应路径

Fig.1 Major reaction branches of ethanol oxidation

部分机理中就不用再包含这些反应了. 这样将提出 的乙醇部分反应机理与 Ra机理整合, 初步得到三组 分燃料的着火反应动力学机理.

\section{2 三组分燃料机理的修正}

使用上述构建的三组分燃料机理分别对异辛
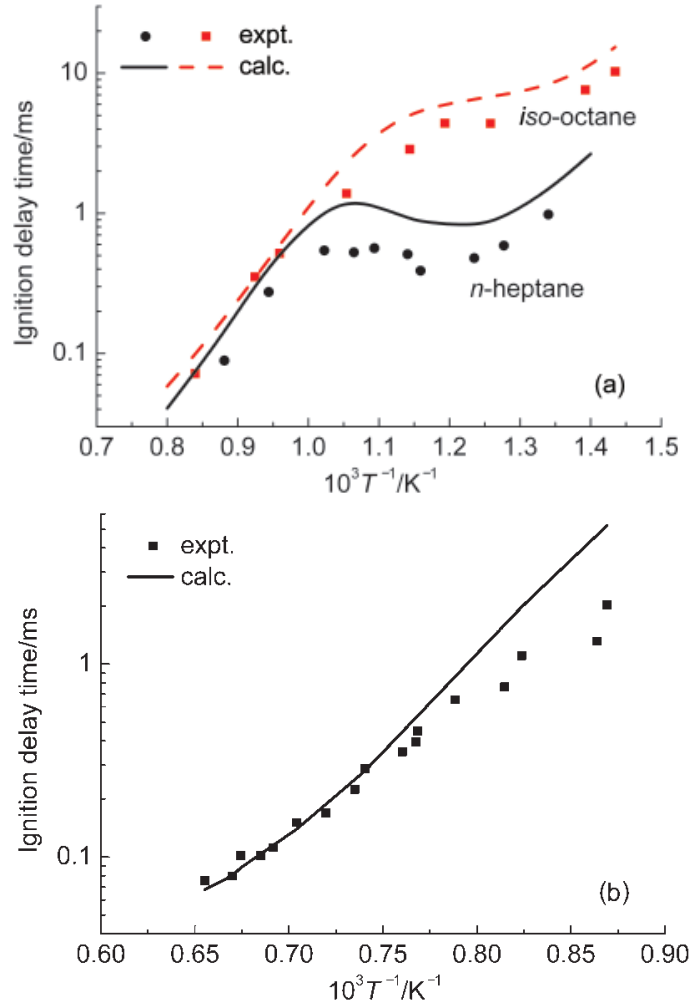

图 2 未修正三组分机理在不同初始温度下计算的着火延 迟时间与实验结果 ${ }^{22,23}$ 比较

Fig.2 Comparison of experimenta ${ }^{22,23}$ and calculated ignition delay time using uncorrected three-component mechanism at different initial temperatures

(a) PRF, conditions: equivalence ratio $=1$ (in air); $p=4.0 \mathrm{MPa}$.

(b) ethanol, conditions: $p=0.35 \mathrm{MPa}$;

volume fractions, $\mathrm{C}_{2} \mathrm{H}_{5} \mathrm{OH} 1.25 \%, \mathrm{O}_{2} 3.75 \%, \mathrm{Ar} 95 \%$
烷、正庚烷和乙醇纯组分燃料的着火延迟时间进行 了初步计算, 计算与实验结果 ${ }^{22,23}$ 对比如图 2 所示. 由图可见, 计算得到的异辛烷、正庚烷和乙醇着火 延迟时间在高温区与实验结果符合较好, 但在低温 区存在较大的差别, 尤其是正庚烷和乙醇. 这说明 无论是基础燃料机理, 还是乙醇机理都存在一定的 缺陷, 因此需要对上述三组分燃料机理进行适当的 修正与调整.

机理修正的基本原则就是调整对低温着火过 程有较大影响, 而对高温过程影响甚微的基元反应. 通常是通过化学反应的灵敏度分析和路径分析来 找到这些基元反应. 对燃料着火时刻进行温度的灵 敏度分析, 分别取灵敏度系数最大的前 10 个反应. 灵敏度系数为正的反应对燃料氧化过程起促进作 用, 灵敏度系数为负的反应对燃料氧化过程起抑制 作用.

首先分析三组分燃料机理中异辛烷和正庚烷 氧化反应路径. 图 3 为异辛烷氧化反应的主要路径. 在低温区异辛烷的主要反应途径如图 3(a)所示. 异 辛烷分子中的氢原子首先被 $\mathrm{OH}$ 基夺走生成异辛烷 基(R2、R3), 接着异辛烷基与氧气加成产生过氧烷 基(R5), 然后过氧烷基与氧气加成产生过氧化氢酮 和 $\mathrm{OH}$ 基(R6), $\mathrm{R} 6$ 生成的 $\mathrm{OH}$ 基又参与 $\mathrm{R} 2$ 反应, 从而 形成由 R2、R3、R5、R6 构成的一个放出大量热量的 低温反应循环. 随着温度的升高, 过氧化氢酮又会 发生分解, 生成 $\mathrm{OH}$ 基(R7), 此时反应系统进入快速 反应阶段. 当系统温度进一步升高, 支链反应 R45 $\left(\mathrm{H}_{2} \mathrm{O}_{2}+\mathrm{M}=\mathrm{OH}+\mathrm{OH}+\mathrm{M}\right)$ 迅速加快, 发生热爆炸反应,

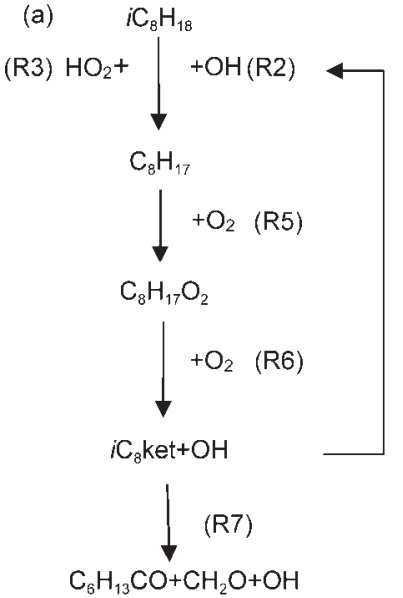

(b)

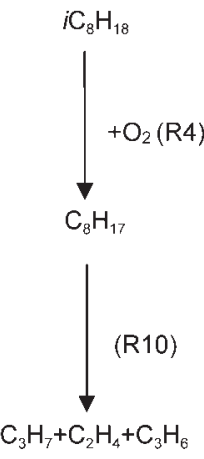

图 3 异辛烷氧化反应路径

Fig.3 Major reaction branches of iso-octane oxidation (a) low temperature, (b) high temperature 
此时系统进入了如图 3(b) 所示的高温反应阶段, 因 此温度迅速升高. 分析这个反应过程, 可以得到影 响异辛烷低温氧化过程的主要基元反应为 R2、R5、 R6 和 R7.

图 4 给出了正庚烷氧化反应的主要路径. 正庚
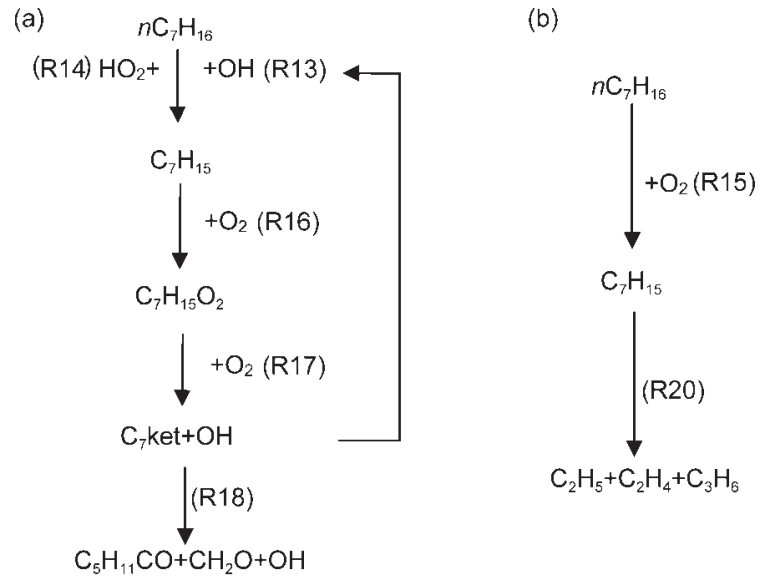

图 4 正庚烷氧化反应路径

Fig.4 Major reaction branches of $\boldsymbol{n}$-heptane oxidation (a) low temperature, (b) high temperature

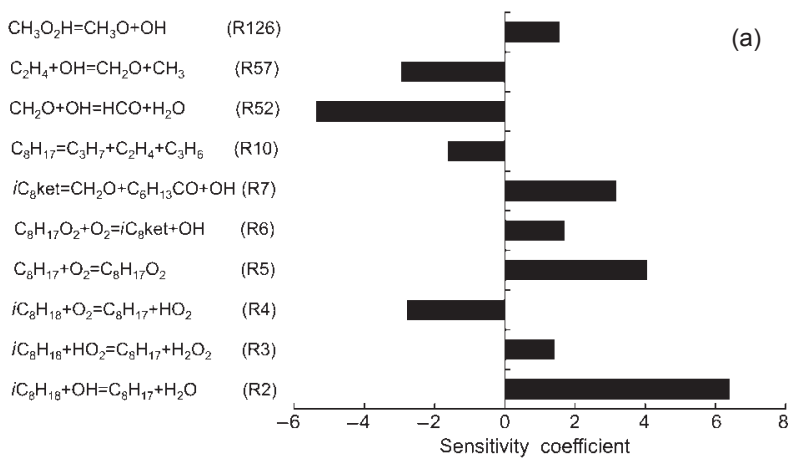

烷氧化反应的主要路径与异辛烷极其相似, 这里就 不再重复描述了. 分析反应过程, 同样可以得到影 响正庚烷低温氧化过程的主要基元反应为 R13、 R16、R17和 R18.

在反应途径分析的基础上, 进一步对异辛烷和 正庚烷在低温 $(700 \mathrm{~K})$ 和高温 $(1250 \mathrm{~K})$ 区的反应进行 温度的灵敏度分析. 图 5 给出了异辛烷在高、低温条 件下的温度灵敏度分析结果. 可以看出, 反应 R5、 R7、R52 对异辛烷低温氧化过程有较大影响, 而对 高温过程影响甚微.

图 6 给出了正庚烷在高、低温条件下的温度灵 敏度分析结果. 由图不难看出, 反应 R17、R18 对正 庚烷低温氧化过程有较大影响, 而对高温过程的影 响较小.

综合上述反应路径分析和温度的灵敏度分析 可得, 对异辛烷而言基元反应 R5 不仅对低温氧化速 度有较大影响, 而且对低温区系统温升也有较大影 响. 对正庚烷而言基元反应 R17、R18 对低温氧化过 程影响较大. 基元反应 R52 对异辛烷和正庚烷低温

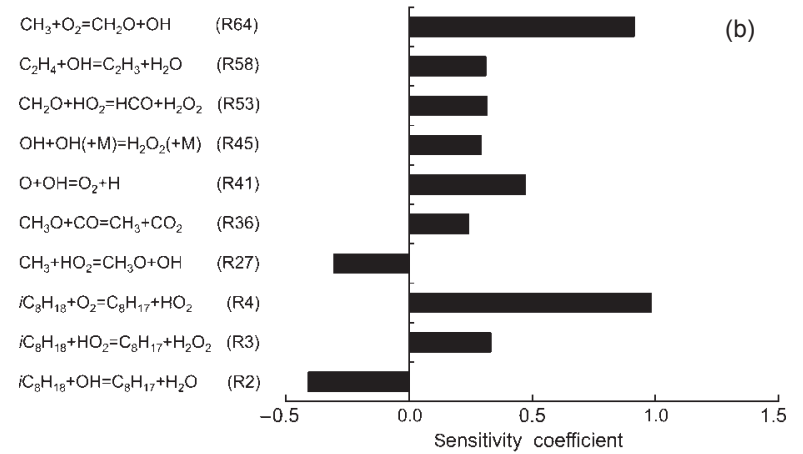

图 5 不同初始温度下异辛烷温度灵敏度系数

Fig.5 iso-Octane sensitivity coefficients at different initial temperatures

(a) low temperature, (b) high temperature
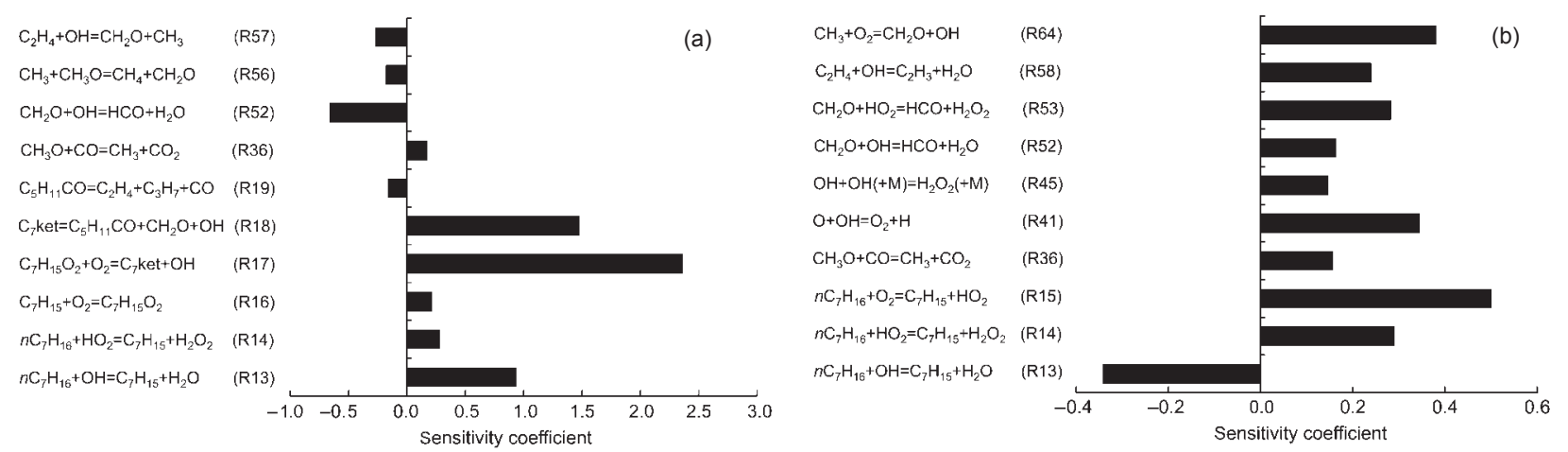

图 6 正庚烷高、低温温度灵敏度分析

Fig.6 $n$-Heptane sensitivity coefficients at different initial temperatures

(a) low temperature, (b) high temperature 
氧化都有很大的负影响. 因此, 对上述四个基元反 应的动力学参数指前因子 $A$ 进行了适当的调整.

基元反应指前因子的调整需要考虑调整方向 (即增大/减小)和调整量. 根据上述分析, R5 和 R52 指前因子的调整标准是调整后的机理能够准确计 算异辛烷着火延迟时间. R5 和 R52 指前因子调整方 向分别是增大和减小, 至于调整量需要经过多次实 验, 才能找到合适调整量. R17和 R18 指前因子调整 的标准是调整后的机理能够准确计算正庚烷着火 延迟时间. R17 和 R18 指前因子调整方向都是增大, 调整量同样需要经过多次试验, 才能找到合适调整 量.

乙醇机理的调整方法与上述基础燃料机理调 整方法一致, 通过路径分析和灵敏度分析找出对低 温着火过程有较大影响而对高温过程影响甚微的 基元反应. 同时为了不影响已经修正的基础燃料机 理, 需选择乙醇氧化为 $\mathrm{C}_{2} \mathrm{H}_{4} 、 \mathrm{CH}_{3} 、 \mathrm{CH}_{2} \mathrm{CO}$ 等小分子 的反应, 并对其动力学参数指前因子进行了适当的 调整. 对异辛烷、正庚烷以及乙醇反应机理进行修 正的基元反应及其动力学参数指前因子如表 3 所 示.

经过上述调整与修改, 三组分燃料反应机理包 含 50 组分和 193 个基元反应(见 Supporing Information). 该机理能够描述异辛烷、正庚烷和乙醇三组分 燃料的着火过程. 使用修正后的机理对异辛烷、正 庚烷和乙醇的着火延迟时间进行了重新计算, 计算 结果与实验结果的对比如图 7 所示, 显然调整之后 的机理能够更准确地预测燃料的着火延迟时间.

\section{3 三组分燃料机理的验证}

验证一个反应动力学机理有效性的重要标志 就是对着火延迟时间的准确预测, 本文详细地比较

表 3 修正的基元反应及其动力学参数指前因子 $A$

Table 3 Modified elementary reaction and kinetic parameter pre-exponential factors $\boldsymbol{A}$

\begin{tabular}{llll}
\hline \multirow{2}{*}{ No. } & \multicolumn{1}{c}{ Reaction } & \multicolumn{2}{c}{$A$} \\
\cline { 3 - 4 } & & previous & modified \\
\hline $\mathrm{R} 5$ & $\mathrm{C}_{8} \mathrm{H}_{17}+\mathrm{O}_{2}=\mathrm{C}_{8} \mathrm{H}_{17} \mathrm{O}_{2}$ & $1.05 \times 10^{11}$ & $1.85 \times 10^{11}$ \\
$\mathrm{R} 17$ & $\mathrm{C}_{7} \mathrm{H}_{15} \mathrm{O}_{2}+\mathrm{O}_{2}=\mathrm{C}$-ket $+\mathrm{OH}$ & $1.52 \times 10^{14}$ & $3.02 \times 10^{14}$ \\
$\mathrm{R} 18$ & $\mathrm{C}_{7} \mathrm{ket}=\mathrm{C}_{5} \mathrm{H}_{11} \mathrm{CO}+\mathrm{CH}_{2} \mathrm{O}+\mathrm{OH}$ & $4.00 \times 10^{14}$ & $7.00 \times 10^{14}$ \\
$\mathrm{R} 52$ & $\mathrm{CH}_{2} \mathrm{O}+\mathrm{OH}=\mathrm{HCO}+\mathrm{H}_{2} \mathrm{O}$ & $5.56 \times 10^{10}$ & $4.86 \times 10^{10}$ \\
$\mathrm{R} 133$ & $\left.\left.\mathrm{C}_{2} \mathrm{H}_{5} \mathrm{OH}+\mathrm{M}\right)=\mathrm{C}_{2} \mathrm{H}_{4}+\mathrm{H}_{2} \mathrm{O}+\mathrm{M}\right)$ & $2.79 \times 10^{13}$ & $9.00 \times 10^{13}$ \\
$\mathrm{R} 145$ & $\mathrm{C}_{2} \mathrm{H}_{5} \mathrm{OH}+\mathrm{CH}_{3}=\mathrm{CH}_{3} \mathrm{CHOH}+\mathrm{CH}_{4}$ & $7.28 \times 10^{2}$ & $6.28 \times 10^{3}$ \\
$\mathrm{R} 147$ & $\mathrm{C}_{2} \mathrm{H}_{5} \mathrm{OH}+\mathrm{HO}_{2}=\mathrm{CH}_{3} \mathrm{CHOH}+\mathrm{H}_{2} \mathrm{O}_{2}$ & $8.80 \times 10^{3}$ & $6.50 \times 10^{4}$ \\
\hline
\end{tabular}
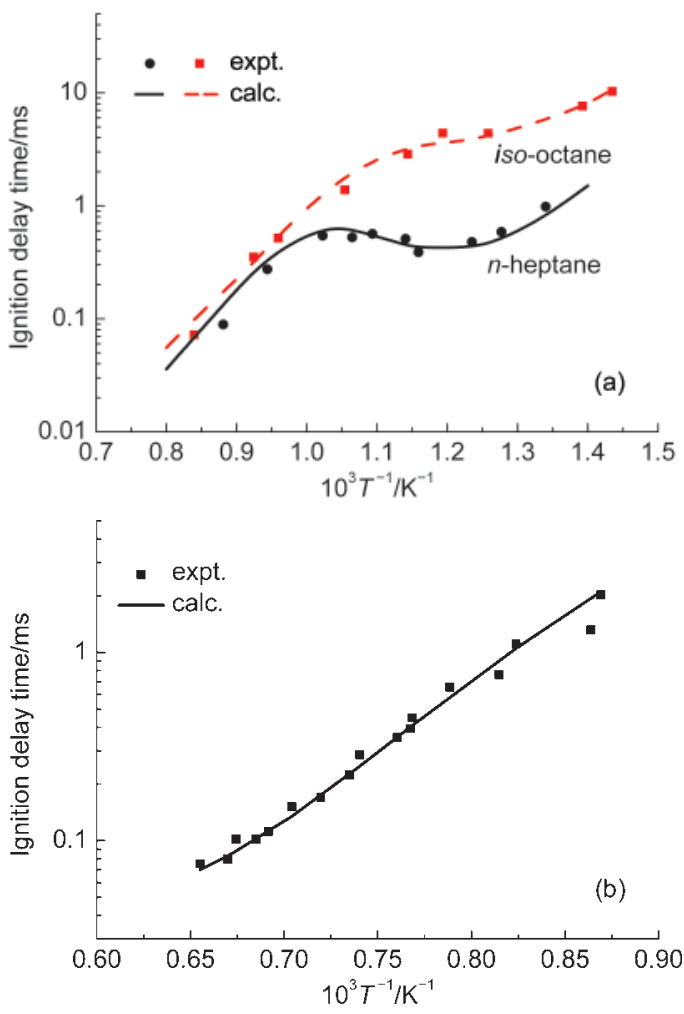

图 7 修正后三组分机理在不同初始温度下计算的 着火延迟时间与实验结果 ${ }^{22,23}$ 比较

Fig.7 Comparison of experimental ${ }^{22,23}$ and calculated ignition delay time using modified three-component mechanism at different initial temperatures

(a) PRF, conditions: equivalence ratio $=1$ (in air); $p=4.0 \mathrm{MPa}$. (b) ethanol, conditions: $p=0.35 \mathrm{MPa}$; volume fractions, $\mathrm{C}_{2} \mathrm{H}_{5} \mathrm{OH} 1.25 \%, \mathrm{O}_{2} 3.75 \%$, Ar 95\%

了单组分、PRF、三组分的着火延迟时间. 计算使用 化学反应动力学程序 CHEMKIN ${ }^{24}$ 为着重研究反应 机理, 计算中均采用零维绝热模型, 忽略流动换热 等因素影响. 用于对比着火延迟时间的实验数据均 来自激波管实验, 在激波管模拟计算中, 假定激波 过后激波管内气体反应在定容、匀质绝热的条件下 进行.

图 8 为初始压力为 $4 \mathrm{MPa}$ 、化学计量比为 1.0 、温 度范围 700-1250 K 时四种基础燃料(异辛烷、正庚 烷、PRF60、PRF80)的着火延迟时间随温度的变化关 系. 实验数据点来自 Fieweger 等 ${ }^{22}$ 的激波管测量结 果. 图中同时给出了使用 Curran 机理 ${ }^{13}$ 和 $\mathrm{Ra}$ 机理 ${ }^{12}$ 计算的结果.

图 8(a)给出了不同温度下三种机理预测的单组 分异辛烷的着火延迟时间. 可以看出本文的三组分 燃料机理、Ra机理和 Curran机理在高温 $(T>1000 \mathrm{~K})$ 条件下的预报值均与实验结果有较高的一致性, 但 

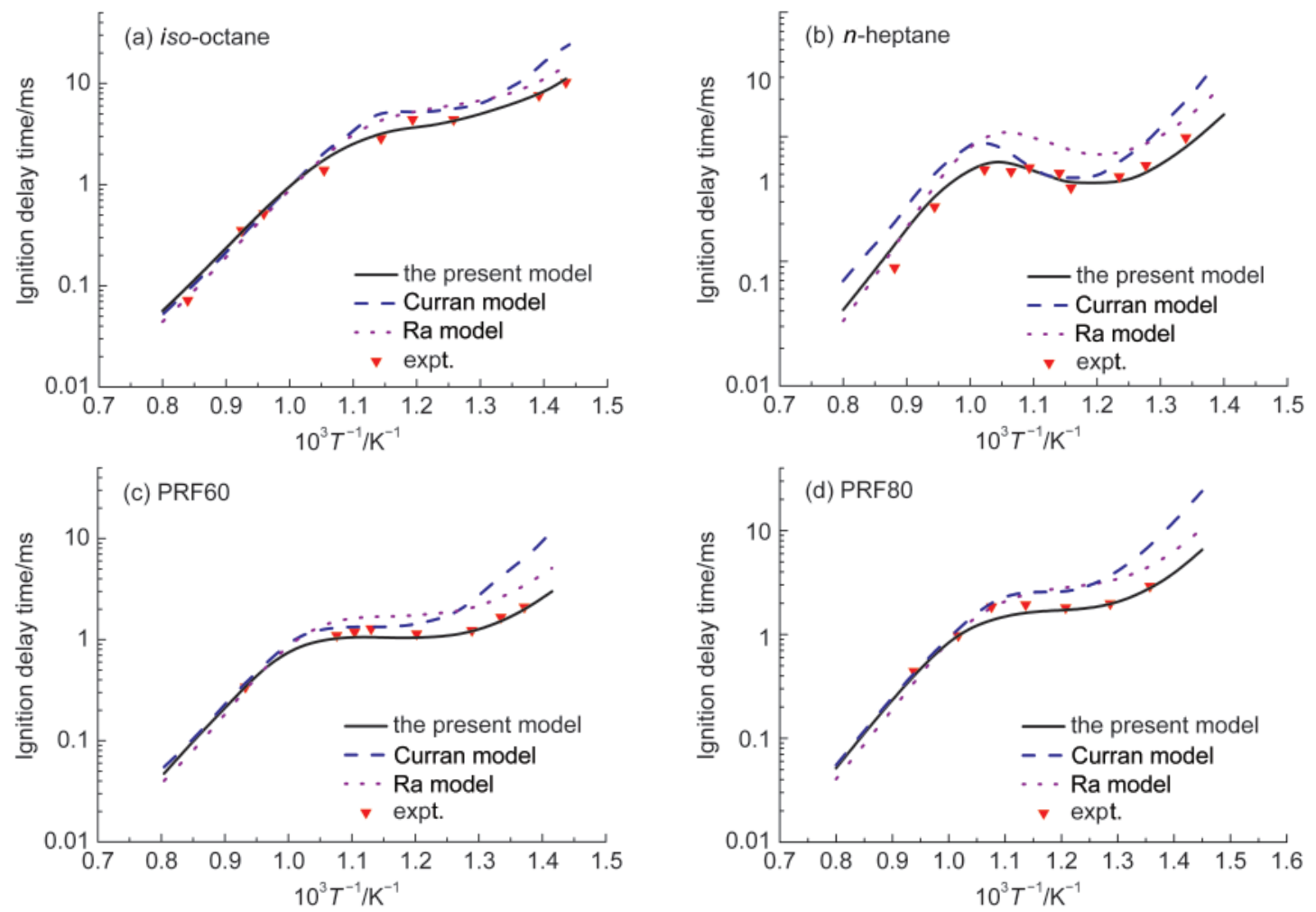

图 8 不同初始温度下计算的基础燃料着火延迟时间与实验结果 ${ }^{22}$ 比较

Fig.8 Comparison of experimental ${ }^{22}$ and calculated PRF ignition delay time at different initial temperatures conditions: equivalence ratio $=1$ (in air); $p=4 \mathrm{MPa}$

在低温 $(T<1000 \mathrm{~K})$ 条件下, Ra机理和 Curran 机理的 预报值明显地高于实验值，而本文的机理与实验结 果非常吻合. 这说明本文的机理在高、低温区间都 能较准确地预测异辛烷着火延迟时间.

图 8(b)给出了不同的温度下三种机理预测单组 分正庚烷的着火延迟时间. 由图可见, Curran机理在 整个温度范围内与实验结果都有较大偏差. Ra机理 和本文机理在高温 $(T>1050 \mathrm{~K})$ 区与实验结果有较好 的一致性, 但在低温 $(T<1050 \mathrm{~K})$ 区 Ra机理明显高估 了实验结果, 而本文机理在整个温度区间都能较好 地预测实验结果, 并准确地预测了 770-950 K 温度 范围内的负温度效应(NTC).

图 8(c,d) 给出了不同温度下三种机理预测 PRF 的着火延迟时间. 由图可以看出, 本文机理的预测 值与实验结果有很好的一致性, 而 Ra机理和 Curran 机理的预测值在低温时与实验结果有较大偏差. 比 较图 8(c, d) 可以得出, PRF 的着火延迟时间随着异 辛烷比例增大而增加, 负温度效应随着异辛烷比例 增大而减小.

图 9 为乙醇着火延迟时间与实验结果的对比. 其中, 图 9(a)给出了三个不同乙醇机理的计算与实 验测量的着火延迟时间随温度的变化关系, 图 9(b)
给出了本文机理在不同化学计量比下计算得到的 乙醇着火延迟时间与实验结果的对比. 图中的实验 数据来自于 Dunphy 和 $\mathrm{Simmi}^{23}$ 在激波管中的测量 结果. 在计算乙醇着火延迟时间时, 由于初始温度 较高, 乙醇绝热燃烧温度在 $2000 \mathrm{~K}$ 左右, 如果还以 温度升高 $400 \mathrm{~K}$ 来计算着火延迟时间, 必然会带来 较大的计算误差. 因此本文将乙醇着火延迟时间定 义为 $\mathrm{OH}$ 基浓度达到最大值所经过的时间. 由图 9(a) 可以看出, 本文机理和 Marinov 详细机理 ${ }^{21}$ 计算的乙 醇着火延迟与实验数据比较接近, 而 Andrae 机理 ${ }^{15}$ 在 1250-1550 K温度范围内低于实验数据, 过早着 火. 图 9(b)表明不同当量比下本文机理仍然能较好 地预测乙醇着火延迟时间.

图 10 给出了压力分别为 $1 、 3$ 和 $5 \mathrm{MPa}$, 化学计 量比为 1 , 液态体积分数为 $20 \%$ 乙醇、 $62 \%$ 异辛烷、 $18 \%$ 正庚烷三组分燃料在 670-1250 K 温度范围内 的着火延迟时间随温度的变化关系. 实验数据来自 Fikri 等 ${ }^{25}$ 在激波管的测量结果. 由图可见, 压力为 1 MPa 时, 本文机理比 Andrae 机理更接近实验结果. 压力增大到 3 和 $5 \mathrm{MPa}$, 本文机理和 Andrae 机理总 体上均能与实验结果一致, 但在高温段 Andrae机理 的计算结果略低于实验结果, 而本文机理无论在高 

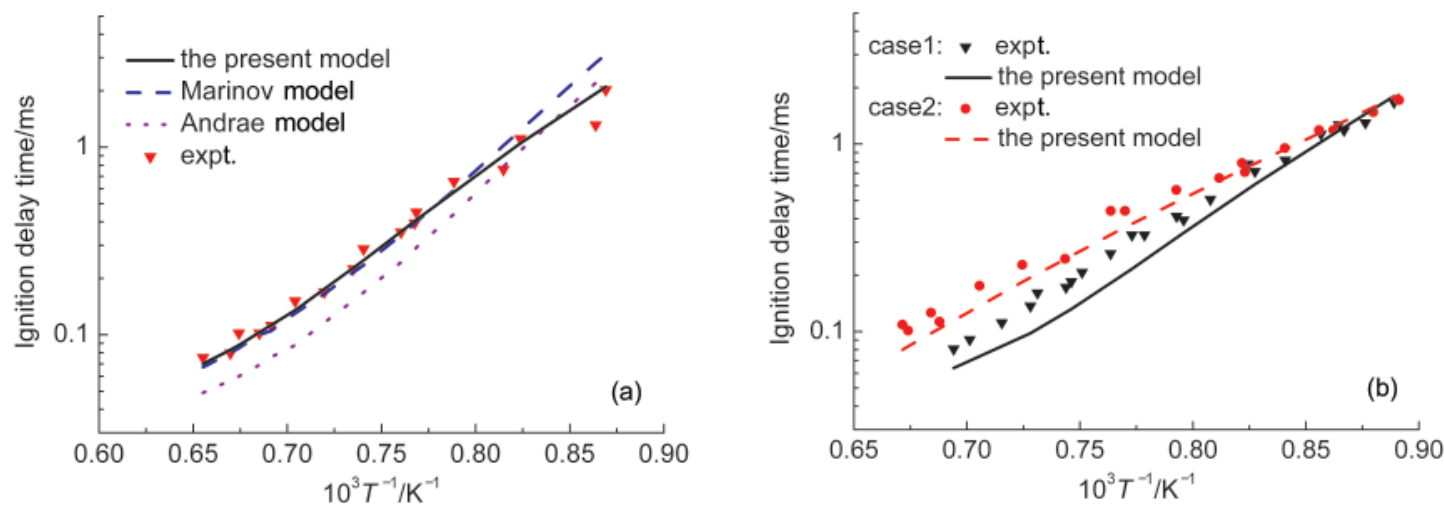

图 9 不同初始温度下计算的乙醇着火延迟与实验结果 ${ }^{23}$ 比较

Fig.9 Comparison of experimental ${ }^{23}$ and calculated ethanol ignition delay time at different temperatures

(a) using various mechanisms, conditions: $p=0.35 \mathrm{MPa}$; volume fractions, $\mathrm{C}_{2} \mathrm{H}_{5} \mathrm{OH} 1.25 \%, \mathrm{O}_{2} 3.75 \%$, $\mathrm{Ar} 95 \%$;

(b) at different equivalence ratios, conditions: (case1) $p=0.34 \mathrm{MPa}$, volume fractions, $\mathrm{C}_{2} \mathrm{H}_{5} \mathrm{OH} 1.25 \%, \mathrm{O}_{2} 7.5 \%, \mathrm{Ar} 91.25 \%$, (case2) $p=0.34 \mathrm{MPa}$, volume fractions, $\mathrm{C}_{2} \mathrm{H}_{5} \mathrm{OH} 2.5 \%, \mathrm{O}_{2} 3.75 \%$, Ar $93.75 \%$

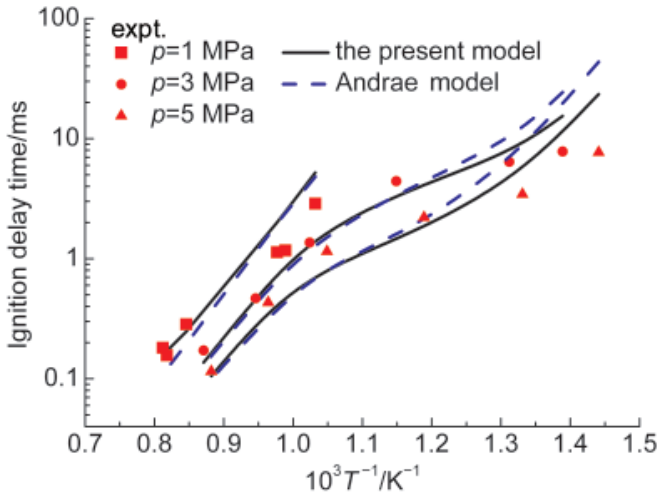

图 10 不同初始温度下计算的三组分燃料着火延迟 时间与实验结果 ${ }^{25}$ 比较

Fig.10 Comparison of experimental ${ }^{25}$ and calculated tertiary mixtures ignition delay time at different initial temperatures

温段还是低温段都比 Andrae机理更准确一些.

\section{4 结 论}

通过反应路径分析和灵敏度分析, 在 $\mathrm{Ra}$ 基础燃 料机理和 Marinov 乙醇机理的基础上, 合理地修正 和补充重要的低温反应, 构建了一个能够准确描述 异辛烷/正庚烷/乙醇三组分燃料着火过程的、包含 50 组分和 193 个基元反应的着火化学动力学机理.

使用本文提出的三组分燃料机理计算单组分 (异辛烷、正庚烷、乙醇)燃料、双组分、PRF 燃料和三 组分燃料的着火延迟时间与实验结果有更好的一 致性. 表明该机理能够更准确地预测异辛烷/正庚 烷/乙醇三组分燃料的着火延迟时间.

提出三组分燃料机理包含较少的组分数与反 应数, 因而可适用汽油掺烧乙醇的多维数值模拟.
Supporting Information Available: Reaction mechanisms of three-component fuel have been included. This information is available free of charge via the internet at http://www.whxb. pku.edu.cn.

\section{References}

(1) Yücesu, H. S.; Topgül, T.; Cinar, C.; Okur, M. Appl. Therm. Eng. 2006, 26 (17-18), 2272.

(2) Maricq, M. M. Combust. Flame 2012, 159 (1), 170. doi: 10.1016/j.combustflame.2011.07.010

(3) Yang, H. H.; Liu, T. C.; Chang, C. F.; Lee, E. Appl. Energy 2012, 89 (1), 281. doi: 10.1016/j.apenergy.2011.07.035

(4) Lemaire, R.; Therssen, E.; Desgroux, P. Fuel 2010, 89 (12), 3952. doi: 10.1016/j.fuel.2010.06.031

(5) Dong, S. R.; Song, C. L.; Zhao, C. P.; Zhang, Y. F.; Guo, Z. P.; Xu, G. P. Journal of Tianjin University 2006, No. 1, 68. [董素 荣, 宋崇林, 赵昌普, 张延峰, 郭振鹏, 徐冠鹏. 天津大学学报, 2006, No. 1, 68.]

(6) Chen, L.; Stone, R.; Richardson, D. Fuel 2012, 96, 120

(7) Zhang, Q. F.; Zheng, C. L.; He, Z. W.; Wang, Y. Transactions of CSICE 2011, No. 2, 133. [张庆峰, 郑朝蕾, 何祖威, 王迎. 内燃机学报, 2011, No. 2, 133.]

(8) Halstead, M. P.; Kirsch, L. J.; Quinn, C. P. Combust. Flame 1977, 30, 45 .

(9) Cox, R. A.; Cole, J. A. Combust. Flame 1985, 60 (2), 109. doi: 10.1016/0010-2180(85)90001-X

(10) Tanaka, S.; Ayala, F.; Keck, J. C. Combust. Flame 2003, 133 (4), 467. doi: 10.1016/S0010-2180(03)00057-9

(11) Tsurushima, T. Proc. Combust. Inst. 2009, 32 (2), 2835. doi: 10.1016/j.proci.2008.06.018

(12) Ra, Y.; Reitz, R. D. Combust. Flame 2008, 155 (4), 713. doi: 10.1016/j.combustflame.2008.05.002

(13) Curran, H. J.; Pitz, W. J.; Westbrook, C. K.; Callahan, G. V.; 
Dryer, F. L. Symposium (International) on Combustion 1998, 27 (1), 379. doi: 10.1016/S0082-0784(98)80426-8

(14) Andrae, J. C. G. Fuel 2008, 87 (10-11), 2013.

(15) Andrae, J. C. G.; Head, R. A. Combust. Flame 2009, 156 (4), 842. doi: 10.1016/j.combustflame.2008.10.002

(16) Andrae, J. C. G.; Björnbom, P.; Cracknell, R. F.; Kalghatgi, G. T. Combust. Flame 2007, 149 (1-2), 2.

(17) Andrae, J. C. G.; Brinck, T.; Kalghatgi, G. T. Combust. Flame 2008, 155 (4), 696. doi: 10.1016/j.combustflame.2008.05.010

(18) Anderlohr, J. M.; Bounaceur, R.; Pires Da Cruz, A.; Battin-Leclerc, F. Combust. Flame 2009, 156 (2), 505. doi: 10.1016/j.combustflame.2008.09.009

(19) Sakai, Y.; Miyoshi, A.; Koshi, M.; Pitz, W. J. Proc. Combust. Inst. 2009, 32 (1), 411. doi: 10.1016/j.proci.2008.06.154

(20) Zhang, Q. F.; Zheng, C. L.; He, Z. W.; Wang, Y. Acta
Phys. -Chim. Sin. 2011, 27, 530. [张庆峰, 郑朝蕾, 何祖威, 王 迎. 物理化学学报, 2011, 27, 530.] doi: 10.3866/PKU. WHXB20110334

(21) Marinov, N. M. Int. J. Chem. Kinet. 1999, 31 (3), 183. doi: 10.1002/(SICI)1097-4601(1999)31:3<183::AID-KIN3>3.0.CO; $2-\mathrm{X}$

(22) Fieweger, K.; Blumenthal, R.; Adomeit, G. Combust. Flame 1997, 109 (4), 599. doi: 10.1016/S0010-2180(97)00049-7

(23) Dunphy, M. P.; Simmie, J. M. Journal of the Chemical Society, Faraday Transactions 1991, 87 (11), 1691. doi: 10.1039/ $\mathrm{ft} 9918701691$

(24) Kee, R. J.; Rupley, F. M.; Miller, J. A.; et al. CHEMKIN Release 4.1; Reaction Design: San Diego, CA, 2006.

(25) Fikri, M.; Herzler, J.; Starke, R.; Schulz, C.; Roth, P.; Kalghatgi, G. T. Combust. Flame 2008, 152 (1-2), 276. 